\title{
GRAVITY WAVES OSCILLATIONS AT SEMICIRCULAR AND GENERAL 2-D CONTAINERS: AN EFFICIENT COMPUTATIONAL APPROACH TO 2-D SLOSHING PROBLEM
}

\author{
M. A. FONTELOS AND J. LÓPEZ-RÍOS
}

\begin{abstract}
We compute the natural frequencies for the oscillations of the free boundary of gravity waves in contact with a solid container. First, we study the case of a semicircular shaped container. We deduce an integrodifferential evolutionary equation for the linearized free boundary and impose pinned-end and free-end boundary conditions. For both cases, the natural oscillations frequencies for the free surfaces are provided and compared with the frequencies in the absence of solid walls. Then we study the effect of having an underwater rectangle shaped bottom in a rectangular container and the corresponding frequencies. The method introduced can be applied to arbitrary 2D containers, with all the information on their geometry contained into a matrix (related to the conformal mapping into a half-plane) that appears as a factor in a linear system for the computation of eigenfrequencies.
\end{abstract}

\section{INTRODUCTION}

The water-waves problem for an ideal liquid consists of describing the motion of a layer of incompressible, inviscid fluid, delimited below by a solid bottom, and above by a free surface under the influence of gravity. It is modeled by means of conservation laws, together with suitable boundary conditions on the surface and bottom [17]. Among the many applications to coastal engineering of the general formulation, we focus on the explicit determination of the wave shape for bounded, simply connected domains in two dimensions.

In this paper we determine the oscillations of the liquid free surface in a two dimensional bounded container, the so called sloshing problem. Due to the scales, surface tension effects are neglected and so, we are dealing with the so-called two dimensional gravity waves on bounded domains. Nevertheless, at this point an important difference with respect to the classical water waves formulation arises: the presence of vertical walls and the contact with

Date: March 21, 2020.

2010 Mathematics Subject Classification. 35Q31; 35J57; $44 \mathrm{~A} 15$.

Key words and phrases. Free boundary problems; water-waves equations; Hilbert transform; conformal mappings.

M. A. Fontelos was supported by xxxx.

J. López-Ríos was supported by xxxx. 
the free surface. We are going to introduce analytical tools to prove that the geometry of the container strongly influences the frequencies. Moreover, we determine such frequencies in various $2 \mathrm{D}$ geometries that can be conformally mapped onto the complex half-plane.

The main strengths of our method to find sloshing frequencies lie in the fact that, by using Tchebyshev polynomials, many computations are explicit and this, of course, provides better precision and faster matrix manipulation. Moreover, frequencies can be computed for arbitrary 2D domains by means of conformal mapping towards a semicircular container. Remarkably, all the information on the domain shape is contained in a single matrix that enters into a product of matrices whose eigenvalues are the sloshing frequencies. This provides a very robust, efficient and general method.

Let $\mathbf{u}(x, y)=(u, v)$ be the velocity of the flow, where $u$ and $v$ are the $x$ and $y$ components of the velocity. Since we are considering an incompressible fluid, we introduce the velocity potential $\varphi(x, y)$ such that $\mathbf{u}=\nabla \varphi$. Then the general water-waves system is given by

$$
\begin{cases}\Delta \varphi=0, & \Omega_{t}, \\ \eta_{t}+\eta_{x} \varphi_{x}=\varphi_{y}, & y=\eta, \\ \varphi_{t}+\frac{1}{2}\left(\varphi_{x}^{2}+\varphi_{y}^{2}\right)+g \eta=0, & y=\eta, \\ \partial_{n} \varphi=0, & y=b,\end{cases}
$$

where $\eta$ and $b$ are the free boundary and bottom parametrization, respectively, $g$ is the acceleration due to gravity and $\Omega_{t}=\left\{(x, y) \in \mathbb{R}^{2}: b(x)<\right.$ $y<\eta(t, x)\}$.

Concerning the evolution of the free surface for non flat bottoms and different geometries in unbounded domains we mention the work by Struik [24] who studied the existence of periodic traveling waves, Nalimov [20], Yosihara [26] and Craig [8] in the two dimensional, small data case, and including some asymptotic regimes, Fontelos et. al $[9,10]$ who studied the inverse problem of detecting bottoms through the measurement of free surfaces and the occurrence of certain specific profiles. We mention the book by Lannes [17] for a general review on well posedness of system (1) within a Sobolev class.

Hereafter $\Omega_{t}$ is a bounded, simply connected domain in $\mathbb{R}^{2}$. To compute explicitly the oscillations we are going to linearize system (1) and restate the problem, through a conformal transplant, in the lower half-plane. By relating the value of the harmonic function $\phi$ and its normal derivative at the free boundary, we get an explicit representation of the DirichletNeumann operator. A related problem, that has been considered recently, is the evolution of the free surface of a perfect incompressible fluid in the presence of capillary forces [15], under the context of the oscillations in a nozzle of an inkjet printer. In that work, the authors consider two different possibilities for the contact line, which have been considered before in the 
literature. The 'pinned-end edge condition' [3, 12], where the contact line is always pinned to the solid surface and the 'free-end condition' where the contact angle between the fluid-air interface and the side walls is fixed and the contact line is allowed to move. The study of oscillations for the capillary case have been studied, asymptotically, in [23] for large Bond numbers. We mention the works by Bostwick and Steen $[4,5]$ where an eigenvalue problem for linear operators is studied, involving various geometries with solid walls.

Following [15], we will consider both the pinned-end boundary condition and the free-end boundary condition, with contact angle $\pi / 2$. We consider standing waves in bounded domains and formulate the problem as an integrodifferential equation on a suitable functional space. By conformal mapping techniques, we compute directly the eigenfrequencies and characterize the terms involving the particular effects of the geometry.

The main motivation of the present work is in relation of controlling the surface gravity waves in a container by injecting jet fluids into the bath inside. In [11], the authors proposed a damped linear gravity waves model, to attenuate the waves in a cooper converter by using triangular finite elements to mesh the bounded domain. Controlling the surface by different methods is of practical interest in oceanography; among others we mention [22], where the authors designed the 'best' moving solid bottom generating a prescribed wave under the context of a BBM type equation and [1] where the local exact controllability of the full water-waves system is obtained by controlling a localized portion of the free surface.

As a consequence of the characterization by conformal mappings, in the last part of the work, we develop an interesting application which has received a lot of attention in these days [7, 17]. Namely, the distribution of rectangular underwater objects and its effect on the free surface. By using our method we were able to compare how the eigenfrequencies are changing in terms of the height and/or the wide of the obstacle.

The rest of the paper is organized as follows. In section 2, we formulate the problem as well as its linearization. Then we introduce conformal mappings that allow to reduce it to the problem in half-space and compute the Dirichlet to Neumann operator for a given geometry. In Section 3 we study the eigenfrequencies of the resulting integrodifferential evolution equation in the case of a container with the geometry of half a circle. We consider the problem in the cases of pinned and free-end conditions for the contact between free boundary and container and, in both cases, separate symmetric and antisymmetric eigenfunctions. Finally, in section 4, we study the problem in a rectangular container with an obstacle by means of a SchwarzChristoffel transformation and discuss its effect on oscillations. We finish with a summary of our main conclusions. 


\section{Problem Formulation}

We consider the half cross-section of a cylindrical container of radius 1 , filled with water, which has its center at the origin, See Figure 1. We use the usual notation $z=x+i y$ for complex numbers with $y$ upward and $x$ rightward. The liquid is assumed to be perfect and incompressible. By assuming the density of the fluid is constant and the surface tension is zero, if $p$ is the pressure inside the fluid, the motion is governed by the incompressible Euler equations:

$$
\begin{aligned}
\nabla \cdot \mathbf{u} & =0 \\
\rho\left[\frac{\partial \mathbf{u}}{\partial t}+(\mathbf{u} \cdot \nabla) \mathbf{u}\right] & =-\nabla p-g e_{2}
\end{aligned}
$$

with $-g e_{2}$ being the constant acceleration of gravity, $g>0$ and $e_{2}$ the unit upward vector in the vertical direction.

By considering the potential function $\varphi$ of $\mathbf{u}$, so that $\mathbf{u}=\nabla \varphi$, we reformulate the problem in terms of $\varphi$ as

$$
\begin{aligned}
\Delta \varphi & =0 \\
\frac{\partial \varphi}{\partial t}+\frac{1}{2}|\nabla \varphi|^{2}+\frac{1}{\rho} p+g y & =\text { const. }
\end{aligned}
$$

We complement system above with the impermeability condition at the solid walls, $\mathbf{u} \cdot n=0$, namely

$$
\frac{\partial \varphi}{\partial n}=0
$$

and the fact that the points of the free boundary are advected by the velocity field, giving us the following kinematic equation for $|x|<1$,

$$
\eta_{t}=\frac{\partial \varphi}{\partial n}
$$

2.1. Linearized equations. Let us consider a small perturbation of the free surface

$$
\eta(t, x)=\epsilon \zeta(t, x) .
$$

Then, if we write the velocity potential as

$$
\varphi=\text { const. }+\epsilon \phi
$$

conditions (2)-(5) in terms of $\phi$, at the first order for $\epsilon<<1$, become

$$
\begin{aligned}
\Delta \phi & =0, \\
\frac{\partial \zeta}{\partial t} & =\frac{\partial \phi}{\partial n}, \quad \text { at } y=0,|x| \leq 1 \\
\frac{\partial \phi}{\partial t}+g \zeta & =0, \quad \text { at } y=0,|x| \leq 1 \\
\frac{\partial \phi}{\partial n} & =0, \quad \text { on the solid walls. }
\end{aligned}
$$



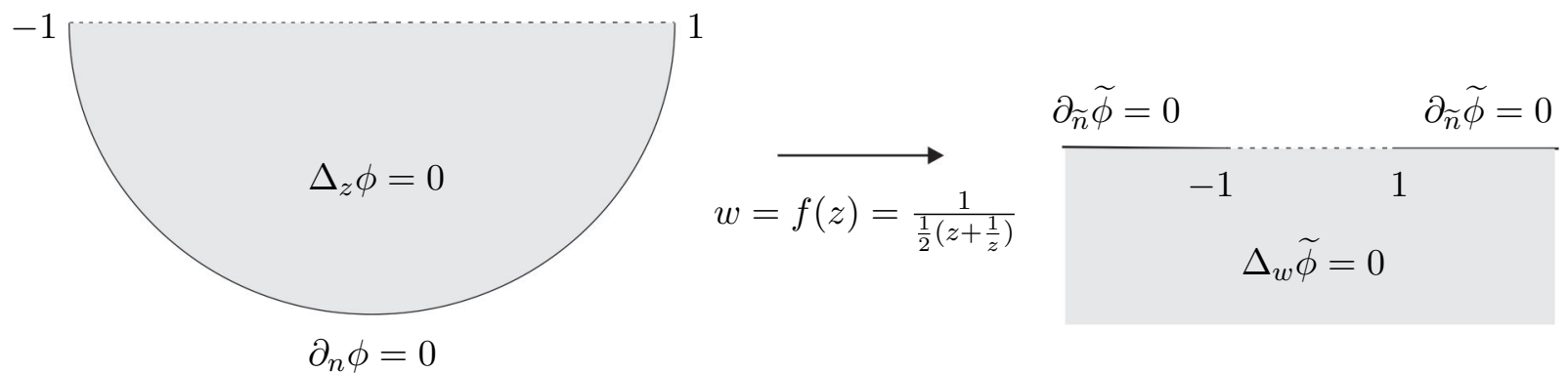

FiguRE 1. Geometry of the problem. The cross section of a half cylinder and the corresponding conditions after mapping into the lower half plane

Therefore, from (7)-(8), on the free boundary (after rescaling to make $g=1)$

$$
\frac{\partial^{2} \phi}{\partial^{2} t}+\frac{\partial \phi}{\partial n}=0
$$

2.2. Conformal transplants. In order to solve system (6)-(9) and more specifically, equation (10), we make a change of variables to transform the half-cylinder into the half-plane, where equation (6) can be solved explicitly. This methodology will allow us to write the Dirichlet-Neumann operator as an integral operator by means of the Hilbert transform. The key idea in this section is an explicit expression of the normal derivative in terms of the conformal map.

Let $\psi$ be a real-valued function written as (see [2], Section 6.5)

$$
\psi:(x, y) \rightarrow \psi(x, y)=\psi(z)
$$

be defined in a domain $D$. Then $\widetilde{\psi}$ is defined in $\widetilde{D}$ as follows: for any $w \in \widetilde{D}$ let

$$
\widetilde{\psi}(w):=\psi\left(f^{[-1]}(w)\right)=\psi\left(x\left(x^{\prime}, y^{\prime}\right), y\left(x^{\prime}, y^{\prime}\right)\right) .
$$

By definition

$$
\psi(x, y)=\widetilde{\psi}\left(x^{\prime}(x, y), y^{\prime}(x, y)\right),
$$

where $x^{\prime}+i y^{\prime}=w=f(z)=f(x+i y)$ and $f$ is holomorphic. If the curve $C$ in the $z$-plane is written $z=z(t)$ we have (see [25])

$$
\frac{\partial \psi}{\partial n}=\frac{1}{\left|\frac{d z}{d t}\right|} \operatorname{Im}\left[\left(\frac{\partial \psi}{\partial x}-i \frac{\partial \psi}{\partial y}\right) \frac{d z}{d t}\right] .
$$

Under the conformal mapping $w=f(z), C$ goes into the curve $C^{*}$ : $w(t)=f(z(t))$. By the chain rule, if $\widetilde{\psi}\left(x^{\prime}, y^{\prime}\right)=\psi\left(x\left(x^{\prime}, y^{\prime}\right), y\left(x^{\prime}, y^{\prime}\right)\right)$,

$$
\left|\frac{d w}{d t}\right| \frac{\partial \widetilde{\psi}}{\partial \widetilde{n}}=\operatorname{Im}\left[\left(\frac{\partial \psi}{\partial x}-i \frac{\partial \psi}{\partial y}\right)\left(\frac{\partial x}{\partial x^{\prime}}-i \frac{\partial x}{\partial y^{\prime}}\right) \frac{d w}{d t}\right] .
$$


Now, $z=f^{[-1]}(w)$ implies $d z=f^{[-1]^{\prime}}(w) d w$, and then by the CauchyRiemann equations

$$
\begin{aligned}
\frac{d z}{d w} & =f^{[-1]^{\prime}}(w)=\frac{\partial f^{[-1]}}{\partial x^{\prime}}(w) \\
& =\frac{\partial x}{\partial x^{\prime}}+i \frac{\partial y}{\partial x^{\prime}} \\
& =\frac{\partial x}{\partial x^{\prime}}-i \frac{\partial x}{\partial y^{\prime}}
\end{aligned}
$$

Then, replacing in (12) and using (11)

$$
\left|\frac{d w}{d t}\right| \frac{\partial \widetilde{\psi}}{\partial \widetilde{n}}=\left|\frac{d z}{d t}\right| \frac{\partial \psi}{\partial n} .
$$

Thus the normal derivative of $\widetilde{\psi}$ on $C^{*}$ is given in terms of the normal derivative of $\psi$ at the corresponding point of $C$ by

$$
\frac{\partial \widetilde{\psi}}{\partial \widetilde{n}}=\left|\frac{d z}{d w}\right| \frac{\partial \psi}{\partial n}=\frac{1}{\left|f^{\prime}(z)\right|} \frac{\partial \psi}{\partial n} .
$$

2.3. Derivation of the integrodifferential equation. Let us transform the half-cylinder into the half-plane by means of the conformal map

$$
w=f(z)=\frac{1}{\frac{1}{2}\left(z+\frac{1}{z}\right)} .
$$

Then, system (6)-(10) in variables $w=x^{\prime}+i y^{\prime}$ becomes (see Figure 1)

$$
\begin{aligned}
\Delta \widetilde{\phi} & =0, \quad \text { for } y^{\prime}<0, \\
\frac{\partial \widetilde{\phi}}{\partial \widetilde{n}} & =0, \quad \text { at } y^{\prime}=0,\left|x^{\prime}\right|>1, \\
\frac{\partial^{2} \widetilde{\phi}}{\partial^{2} t}+\frac{\partial \widetilde{\phi}}{\partial \widetilde{n}} & =0, \quad \text { at } y^{\prime}=0,\left|x^{\prime}\right| \leq 1,
\end{aligned}
$$

together with the following conditions for the asymptotic behavior at infinity:

$$
\frac{\partial \phi}{\partial x^{\prime}}, \frac{\partial \phi}{\partial y^{\prime}} \rightarrow 0, \text { as } y^{\prime} \rightarrow-\infty \text { or }\left|x^{\prime}\right| \rightarrow \infty
$$

By taking Fourier transform in $x^{\prime}$ of $(15)$, denoted by $\widetilde{\Phi}$, we have

$$
\frac{d^{2} \widetilde{\Phi}}{d^{2} y^{\prime}}-k^{2} \widetilde{\Phi}=0
$$

which implies, by the asymptotic behavior $y^{\prime} \rightarrow-\infty$,

$$
\widetilde{\Phi}\left(t, k, y^{\prime}\right)=\widetilde{\Phi}(t, k, 0) e^{|k| y^{\prime}} .
$$


By taking inverse Fourier transform

$$
\widetilde{\phi}\left(t, x^{\prime}, y^{\prime}\right)=-\frac{y^{\prime}}{\pi} \int_{-\infty}^{+\infty} \frac{\widetilde{\phi}(t, \zeta, 0)}{\left(x^{\prime}-\zeta\right)^{2}+y^{\prime}} d \zeta .
$$

Moreover, if we take $y^{\prime}$ derivative in (18) and evaluate at $y^{\prime}=0$ we find

$$
\left.\frac{\partial \widetilde{\phi}}{\partial y^{\prime}}\right|_{y^{\prime}=0}=\frac{1}{\pi} P . V \int_{-\infty}^{+\infty} \frac{\partial \widetilde{\phi} / \partial x^{\prime}(t, \zeta, 0)}{x^{\prime}-\zeta} d \zeta=H\left(\left.\frac{\partial \widetilde{\phi}}{\partial x^{\prime}}\right|_{y^{\prime}=0}\right),
$$

where $H$ is the Hilbert transform (see [14]). Equation (19) provide us with an explicit representation of the Dirichlet-Neumann operator in terms of the Hilbert transform, a linear operator of the derivative of $\widetilde{\phi}$.

By using the fact that $H H=-I$ (see [14]) we have

$$
\left.\frac{\partial \widetilde{\phi}}{\partial x^{\prime}}\right|_{y^{\prime}=0}=-\frac{1}{\pi} P . V \int_{-1}^{1} \frac{\partial \widetilde{\phi} / \partial y^{\prime}(t, \zeta, 0)}{x^{\prime}-\zeta} d \zeta,
$$

which is the integrodifferential representation of the Dirichlet-Neumann operator we were looking for.

\section{Solution OF the integrodifferential EQUATION FOr $\widetilde{\phi}$}

In this section, we use the method of separation of variables to rewrite equation (20) as an eigenvalue problem for an eigenvalue $\lambda$, and the corresponding eigenfunction in the variable $x^{\prime}$. This is possible by the linearization (10) assumed in subsection 2.1. Then the Tchebyshev polynomial base will be use to decompose the spatial frequencies together with an approximation by a matrix problem of finite dimension, which approximate eigenvalues $\lambda$ at any order.

From (10) and (14) we have

$$
\frac{\partial^{2} \widetilde{\phi}}{\partial^{2} t}+\left|f^{\prime}(z)\right| \frac{\partial \widetilde{\phi}}{\partial \widetilde{n}}=0
$$

Since $\frac{\partial \widetilde{\phi}}{\partial y^{\prime}}=\frac{\partial \widetilde{\phi}}{\partial \widetilde{n}}$ for $x^{\prime} \in[-1,1],(20)$ can be written as

$$
\begin{aligned}
\left.\frac{\partial \widetilde{\phi}}{\partial x^{\prime}}\right|_{y^{\prime}=0} & =-\frac{1}{\pi} P \cdot V \int_{-1}^{1} \frac{\frac{\partial \widetilde{\phi}}{\partial \tilde{n}}(t, \zeta, 0)}{x^{\prime}-\zeta} d \zeta \\
& =\frac{1}{\pi} P . V \int_{-1}^{1} \frac{\frac{\partial^{2} \widetilde{\phi}}{\partial^{2} t}(t, \zeta, 0)}{\left|f^{\prime}(\zeta)\right|\left(x^{\prime}-\zeta\right)} d \zeta .
\end{aligned}
$$

To solve this integrodifferential equation, by the separation of variables method, let $\widetilde{\phi}\left(t, x^{\prime}, 0\right)=A(t) S\left(x^{\prime}\right)$. Then, by the last equation,

$$
A(t) S^{\prime}\left(x^{\prime}\right)=\frac{1}{\pi} P . V \int_{-1}^{1} \frac{A^{\prime \prime}(t) S(\zeta)}{\left|f^{\prime}(\zeta)\right|\left(x^{\prime}-\zeta\right)} d \zeta
$$


which is equivalent to the eigenvalue problem

$$
A^{\prime \prime}+\lambda A=0,
$$

with $\lambda \in \mathbb{R}$ such that

$$
S^{\prime}\left(x^{\prime}\right)=-\frac{\lambda}{\pi} P . V \int_{-1}^{1} \frac{S(\zeta)}{\left|f^{\prime}(\zeta)\right|\left(x^{\prime}-\zeta\right)} d \zeta
$$

This equation can be viewed as an integral equation for $S\left(x^{\prime}\right)$ in terms of $S^{\prime}\left(x^{\prime}\right)$ (see Hochstadt, [14]), given by

$$
\lambda \frac{S\left(x^{\prime}\right)}{\left|f^{\prime}(x)\right|}=\frac{1}{\pi} \sqrt{1-x^{\prime 2}} P . V \int_{-1}^{1} \frac{1}{\sqrt{1-\zeta^{2}}} \frac{S^{\prime}(\zeta)}{x^{\prime}-\zeta} d \zeta .
$$

Namely,

$$
\lambda S\left(x^{\prime}\right)=\frac{1}{\pi} \sqrt{1-x^{\prime 2}}\left|f^{\prime}\left(x^{\prime}\right)\right| P . V \int_{-1}^{1} \frac{1}{\sqrt{1-\zeta^{2}}} \frac{S^{\prime}(\zeta)}{x^{\prime}-\zeta} d \zeta
$$

is our eigenvalue problem in the semi-plane in terms of $S$, related to any bounded simply connected domain in the plane through $f(z)$. Note the arising of the term involving the geometry of the problem, represented by the conformal map $f$.

The inversion formula for the Hilbert transform in (22) leading to (23) can also be deduced by taking into account the following: let $T_{r}, U_{r}$, with $r \in \mathbb{N} \cup\{0\}$, be the Tchebyshev polynomials of the first and second kind respectively. Then, for $r \geq 1$,

$$
\pi T_{r}\left(x^{\prime}\right)=P . V \int_{-1}^{1} \frac{\sqrt{1-\zeta^{2}} U_{r-1}(\zeta)}{x^{\prime}-\zeta} d \zeta
$$

and

$$
\frac{1}{\pi} P . V \int_{-1}^{1} \frac{T_{r}(\zeta)}{\sqrt{1-\zeta^{2}}\left(x^{\prime}-\zeta\right)} d \zeta=-U_{r-1}\left(x^{\prime}\right) .
$$

Note that, for any $r \geq 1$,

$$
-T_{r}\left(x^{\prime}\right)=\frac{1}{\pi} P . V \int_{-1}^{1} \frac{\sqrt{1-\zeta^{2}}}{x^{\prime}-\zeta}\left[\frac{1}{\pi} P . V \int_{-1}^{1} \frac{T_{r}\left(\zeta^{\prime}\right)}{\sqrt{1-\zeta^{\prime 2}}\left(\zeta-\zeta^{\prime}\right)} d \zeta^{\prime}\right] d \zeta,
$$

so that for any linear combination $f\left(x^{\prime}\right)$ of $T_{r}\left(x^{\prime}\right), r \geq 1$, we have

$$
-f=\frac{1}{\pi} P . V \int_{-1}^{1} \frac{\sqrt{1-\zeta^{2}}}{x^{\prime}-\zeta}\left[\frac{1}{\pi} P . V \int_{-1}^{1} \frac{f}{\sqrt{1-\zeta^{\prime 2}}\left(\zeta-\zeta^{\prime}\right)} d \zeta^{\prime}\right] d \zeta .
$$

For the sake of accuracy, taking into account that the polynomials $T_{r}(x)$ form an orthogonal basis in $L^{2}\left([-1,1], 1 / \sqrt{1-x^{\prime 2}}\right)$, we assume that

$$
\|f\|_{L^{2}\left([-1,1], 1 / \sqrt{1-x^{\prime 2}}\right)}^{2}=\int_{-1}^{1} f^{2}\left(x^{\prime}\right) \frac{d x^{\prime}}{\sqrt{1-x^{\prime 2}}}<\infty .
$$

The fact that the polynomial $T_{0}\left(x^{\prime}\right)$ is excluded from the linear combination implies the condition $\left(f, T_{0}\right)_{L^{2}\left([-1,1], 1 / \sqrt{1-x^{\prime 2}}\right)}=\frac{2}{\pi} \int_{-1}^{1} \frac{f\left(x^{\prime}\right)}{\sqrt{1-x^{\prime 2}}} d x^{\prime}=$ 
0, a fact that also formally follows by multiplying both sides of (22) by $1 / \sqrt{1-x^{2}}$, integrating, exchanging integrals at the right hand side and using P.V $\int_{-1}^{1} \frac{1}{x^{\prime}-\zeta} \frac{d x^{\prime}}{\sqrt{1-x^{\prime 2}}}=0$. Hence, a solution to (22) with $\lambda \neq 0$ such that $S^{\prime} \in L^{2}\left([-1,1], 1 / \sqrt{1-x^{\prime 2}}\right)$ satisfies

$$
\int_{-1}^{1} \frac{S^{\prime}(x)}{\sqrt{1-x^{2}}} d x=0
$$

and is equivalent to a solution of (24) satisfying in addition

$$
S /|f| \in L^{2}\left([-1,1], 1 / \sqrt{1-x^{2}}\right) .
$$

Let us write the eigenvalue problem for the particular case of the semiplane. By (14) we know that $\frac{\partial \phi}{\partial n}=\left|f^{\prime}(z)\right| \frac{\partial \widetilde{\phi}}{\partial \widetilde{n}}=\left|\frac{\partial x^{\prime}}{\partial x}+i \frac{\partial y^{\prime}}{\partial x}\right| \frac{\partial \widetilde{\phi}}{\partial \widetilde{n}}$.

Now, by definition

$$
x^{\prime}+i y^{\prime}=f(z)=\frac{2}{z+\frac{1}{z}} .
$$

Then, if $x \in[-1,1]$ and $y=0$ we have $x^{\prime} \in[-1,1], y^{\prime}=0$ and thus

$$
x^{\prime}=\frac{2}{x+\frac{1}{x}} \text {. }
$$

From this last equation we get

$$
\frac{1}{x^{2}}+1=\frac{2}{x x^{\prime}}
$$

and since $f(0)=0$

$$
x x^{\prime}=1-\sqrt{1-x^{\prime 2}} .
$$

Therefore,

$$
\begin{aligned}
\left|f^{\prime}(z)\right|=\frac{d x^{\prime}}{d x} & =2 \frac{1-x^{2}}{\left(1+x^{2}\right)^{2}}=\frac{1}{2} x^{\prime 2}\left(\frac{1}{x^{2}}-1\right) \\
& =x^{\prime 2}\left(\frac{1}{x x^{\prime}}-1\right) \\
& =x^{\prime 2}\left(\frac{1}{1-\sqrt{1-x^{\prime 2}}}-1\right) \\
& =\sqrt{1-x^{\prime 2}}\left(1+\sqrt{1-x^{\prime 2}}\right) .
\end{aligned}
$$

Then we obtain the following relation between the normal derivatives in the corresponding domains:

$$
\frac{\partial \phi}{\partial n}=\sqrt{1-x^{\prime 2}}\left(1+\sqrt{1-x^{\prime 2}}\right) \frac{\partial \widetilde{\phi}}{\partial \widetilde{n}} .
$$

By (24) and (30), we have that the eigenvalue problem in the semi-circular container in terms of $S$ is given by

$$
\lambda S\left(x^{\prime}\right)=\frac{1}{\pi}\left(1-x^{\prime 2}\right)\left(1+\sqrt{1-x^{\prime 2}}\right) P . V \int_{-1}^{1} \frac{1}{\sqrt{1-\zeta^{2}}} \frac{S^{\prime}(\zeta)}{x^{\prime}-\zeta} d \zeta .
$$


In the next subsections we are going to consider the different possibilities for the contact line at the boundary. Since any general function $S\left(x^{\prime}\right)$ can be decomposed as the sum of an antisymmetric function and a symmetric function, using the Fourier series expansion, if we assume the free-end boundary condition, with contact angle $\pi / 2, S\left(x^{\prime}\right)$ can be expanded in the form

$$
S\left(x^{\prime}\right)=\sum_{n \geq 1} a_{n} \sin \left(\left(n-\frac{1}{2}\right) \pi x^{\prime}\right), \quad \text { or } \quad S\left(x^{\prime}\right)=\sum_{n \geq 1} b_{n} \cos \left(n \pi x^{\prime}\right) .
$$

In the same way, if we assume the pinned-end boundary condition, $S( \pm 1)=$ $0, S\left(x^{\prime}\right)$ can be expanded in the form

$$
S\left(x^{\prime}\right)=\sum_{n \geq 1} \widetilde{a}_{n} \sin \left(n \pi x^{\prime}\right), \quad \text { or } \quad S\left(x^{\prime}\right)=\sum_{n \geq 1} \widetilde{b}_{n} \cos \left(\left(n-\frac{1}{2}\right) \pi x^{\prime}\right) .
$$

3.1. Antisymmetric solution with respect to $x^{\prime}=0$, the free-end case. As we mentioned before, since the eigenvalue problem relates the Hilbert transform restricted to the interval $[-1,1]$, a suitable base to expand the solutions in series is the one given by the Tchebyshev polynomials (see [19]).

Let $S\left(x^{\prime}\right)=\sum_{n \geq 1} a_{n} \sin \left(\left(n-\frac{1}{2}\right) \pi x^{\prime}\right)$ and the Tchebyshev polynomial expansions:

$$
\begin{aligned}
& \cos \left(\left(n-\frac{1}{2}\right) \pi x^{\prime}\right)=\sum_{k \geq 0} c_{k n} T_{k}\left(x^{\prime}\right), \\
& \sin \left(\left(n-\frac{1}{2}\right) \pi x^{\prime}\right)=\sum_{r \geq 1} e_{r n} \sqrt{1-x^{\prime 2}} U_{r-1}\left(x^{\prime}\right),
\end{aligned}
$$

in such a way that

$$
S\left(x^{\prime}\right)=\sum_{n, r} e_{r n} a_{n} \sqrt{1-x^{\prime 2}} U_{r-1}\left(x^{\prime}\right)
$$

By the orthogonality of the Tchebyshev polynomials,

$$
\begin{gathered}
c_{k n}= \begin{cases}\frac{2}{\pi} \int_{-1}^{1} \cos \left(\left(n-\frac{1}{2}\right) \pi x^{\prime}\right) \frac{T_{k}\left(x^{\prime}\right)}{\sqrt{1-x^{\prime 2}}} d x^{\prime}, & k \geq 1, \\
\frac{1}{\pi} \int_{-1}^{1} \cos \left(\left(n-\frac{1}{2}\right) \pi x^{\prime}\right) \frac{1}{\sqrt{1-x^{\prime 2}}} d x^{\prime}, & k=0,\end{cases} \\
e_{r n}=\frac{2}{\pi} \int_{-1}^{1} \sin \left(\left(n-\frac{1}{2}\right) \pi x^{\prime}\right) U_{r-1}\left(x^{\prime}\right) d x^{\prime} .
\end{gathered}
$$


Therefore, replacing (34) into (31) and using (26)

$$
\begin{aligned}
\lambda & \sum_{n, r} e_{r n} a_{n} \sqrt{1-x^{\prime 2}} U_{r-1}\left(x^{\prime}\right) \\
& =\frac{1}{\pi}\left(1-x^{\prime 2}\right)\left(1+\sqrt{1-x^{\prime 2}}\right) P . V \int_{-1}^{1} \frac{\sum_{n}\left(n-\frac{1}{2}\right) \pi a_{n} \cos \left(\left(n-\frac{1}{2}\right) \pi \zeta\right)}{\sqrt{1-\zeta^{2}}\left(x^{\prime}-\zeta\right)} d \zeta \\
& =\frac{1}{\pi}\left(1-x^{\prime 2}\right)\left(1+\sqrt{1-x^{\prime 2}}\right) P . V \int_{-1}^{1} \frac{\sum_{n, k}\left(n-\frac{1}{2}\right) \pi c_{k n} a_{n} T_{k}(\zeta)}{\sqrt{1-\zeta^{2}}\left(x^{\prime}-\zeta\right)} d \zeta \\
& =-\left(1-x^{\prime 2}\right)\left(1+\sqrt{1-x^{\prime 2}}\right) \sum_{n, k}\left(n-\frac{1}{2}\right) \pi c_{k n} a_{n}\left(-\frac{1}{\pi} P . V \int_{-1}^{1} \frac{T_{k}(\zeta)}{\sqrt{1-\zeta^{2}}\left(x^{\prime}-\zeta\right)} d \zeta\right) \\
& =-\left(1-x^{\prime 2}\right)\left(1+\sqrt{1-x^{\prime 2}}\right) \sum_{n, k}\left(n-\frac{1}{2}\right) \pi c_{k n} a_{n} U_{k-1}\left(x^{\prime}\right) .
\end{aligned}
$$

Note that from this last step we are assuming that the equality is true for $k \geq 1$, even though $k \geq 0$ in equation (32). Namely, for all $n, r, k \geq 1$

$$
-\lambda \sum_{n, r} e_{r n} a_{n} \frac{U_{r-1}\left(x^{\prime}\right)}{\sqrt{1-x^{\prime 2}}\left(1+\sqrt{1-x^{\prime 2}}\right)}=\sum_{n, k}\left(n-\frac{1}{2}\right) \pi c_{k n} a_{n} U_{k-1}\left(x^{\prime}\right) .
$$

Then, if we write

$$
\frac{U_{r-1}\left(x^{\prime}\right)}{\sqrt{1-x^{\prime 2}}\left(1+\sqrt{1-x^{\prime 2}}\right)}=\sum_{k} d_{k r} U_{k-1}\left(x^{\prime}\right)
$$

for some $d_{k r}$ and we replace in (37):

$$
-\lambda \sum_{n, k, r} d_{k r} e_{r n} a_{n} U_{k-1}\left(x^{\prime}\right)=\sum_{n, k}\left(n-\frac{1}{2}\right) \pi c_{k n} a_{n} U_{k-1}\left(x^{\prime}\right) .
$$

Notice that matrix $D$ contains the information on the geometry of the container; a semicircle in this case.

Thus we get the eigenvalue problem

$$
-\lambda \sum_{n, r} d_{k r} e_{r n} a_{n}=\sum_{n}\left(n-\frac{1}{2}\right) \pi c_{k n} a_{n}
$$

with $c_{k n}, e_{r n}, d_{k r}$ as in (35), (36), (38) respectively and $n, r, k \geq 1$. In matrix form, (39) can be written as

$$
-\lambda D E \mathbf{a}=\pi C\left[\left(i-\frac{1}{2}\right) \delta_{i j}\right] \mathbf{a},
$$

with $D=\left(d_{k r}\right), E=\left(e_{r n}\right), C=\left(c_{k n}\right), \mathbf{a}=\left(a_{n}\right)^{T}$ and we are using $\left[\left(i-\frac{1}{2}\right) \delta_{i j}\right]$ to denote an infinite diagonal matrix with $i$-th element $i-\frac{1}{2}, i=$ $1,2, \ldots$ 
By (38) and the orthogonality of Tchebyshev polynomials we have

$$
\begin{aligned}
d_{k r} & =\frac{2}{\pi} \int_{-1}^{1} \frac{U_{k-1}\left(x^{\prime}\right) U_{r-1}\left(x^{\prime}\right)}{1+\sqrt{1-x^{\prime 2}}} d x^{\prime} \\
& =\frac{2}{\pi} \sum_{i=0}^{r-1} \int_{-1}^{1} \frac{U_{k-r+2 i}\left(x^{\prime}\right)}{1+\sqrt{1-x^{\prime 2}}} d x^{\prime}, \quad k \geq r .
\end{aligned}
$$

What we are going to do next, is to compute an inverse $F$, of matrix $C$, to rewrite equation (40) in a way we can compare the frequencies $\pi\left(i-\frac{1}{2}\right)$, $i=1,2, \ldots$, for gravity waves on the half plane, with the eigenvalues of the matrix $F D E$ ( $F$ to be defined below) arising from the particular geometry of the bounded domain.

Let

$$
f_{k n}=\int_{-1}^{1} \cos \left(\left(n-\frac{1}{2}\right) \pi x^{\prime}\right) T_{k}\left(x^{\prime}\right) d x^{\prime}, \quad k \geq 0, n \geq 1
$$

and assume $\cos \left(\left(n-\frac{1}{2}\right) \pi x^{\prime}\right)=\sum_{k} \alpha_{k n} \frac{T_{k}\left(x^{\prime}\right)}{\sqrt{1-x^{\prime 2}}}$. By the orthogonality of the Tchebyshev polynomials,

$$
\begin{aligned}
\alpha_{k n} & =\left\{\begin{array}{l}
\frac{2}{\pi} \int_{-1}^{1} \cos \left(\left(n-\frac{1}{2}\right) \pi x^{\prime}\right) T_{k}\left(x^{\prime}\right), \quad k \geq 1, \\
\frac{1}{\pi} \int_{-1}^{1} \cos \left(\left(n-\frac{1}{2}\right) \pi x^{\prime}\right), \quad k=0,
\end{array}\right. \\
& = \begin{cases}\frac{2}{\pi} f_{k n}, & k \geq 1, \\
\frac{1}{\pi} f_{k n}, & k=0 .\end{cases}
\end{aligned}
$$

Therefore, by (32)

$$
\begin{aligned}
\delta_{m n} & =\int_{-1}^{1} \cos \left(\left(n-\frac{1}{2}\right) \pi x^{\prime}\right) \cos \left(\left(m-\frac{1}{2}\right) \pi x^{\prime}\right) d x^{\prime} \\
& =\int_{-1}^{1} \sum_{k} c_{k n} T_{k}\left(x^{\prime}\right) \sum_{k^{\prime}} \alpha_{k^{\prime} m} \frac{T_{k^{\prime}}\left(x^{\prime}\right)}{\sqrt{1-x^{\prime 2}}} d x^{\prime} \\
& =\frac{2}{\pi} \int_{-1}^{1} \sum_{k} c_{k n} T_{k}\left(x^{\prime}\right) \sum_{k^{\prime}} f_{k^{\prime} m} \frac{T_{k^{\prime}}\left(x^{\prime}\right)}{\sqrt{1-x^{\prime 2}}} d x^{\prime}+\frac{1}{\pi} c_{0 n} f_{0 m} \int_{-1}^{1} \frac{1}{\sqrt{1-x^{\prime 2}}} d x^{\prime} \\
& =\sum_{k, k^{\prime} \geq 1} f_{k^{\prime} m} c_{k n} \frac{2}{\pi} \int_{-1}^{1} \frac{T_{k}\left(x^{\prime}\right) T_{k^{\prime}}\left(x^{\prime}\right)}{\sqrt{1-x^{\prime 2}}} d x^{\prime}+f_{0 m} c_{0 n} \\
& =\sum_{k \geq 0} f_{k m} c_{k n} .
\end{aligned}
$$

Namely,

$$
F^{T} C=I
$$

with $F=\left(f_{k n}\right)$ given by (41).

Now, before multiplying (40) by $F$, note that $k \geq 0$ and equation (40) is true for $k \geq 1$. Therefore we have to augment the right hand side of (40) 


\begin{tabular}{lccccr}
\hline- & $\lambda_{1}=1.363$ & $\lambda_{2}=4.681$ & $\lambda_{3}=7.886$ & $\lambda_{4}=11.096$ & $\lambda_{5}=14.325$ \\
\hline$a_{1}$ & $9.81 \mathrm{E}-01$ & $7.70 \mathrm{E}-01$ & $1.39 \mathrm{E}-01$ & $4.48 \mathrm{E}-02$ & $-6.98 \mathrm{E}-03$ \\
$a_{2}$ & $-1.70 \mathrm{E}-01$ & $5.03 \mathrm{E}-01$ & $9.30 \mathrm{E}-01$ & $4.94 \mathrm{E}-01$ & $1.91 \mathrm{E}-01$ \\
$a_{3}$ & $7.24 \mathrm{E}-02$ & $-2.92 \mathrm{E}-01$ & $2.34 \mathrm{E}-03$ & $7.62 \mathrm{E}-01$ & $7.50 \mathrm{E}-01$ \\
$a_{4}$ & $-4.07 \mathrm{E}-02$ & $1.84 \mathrm{E}-01$ & $-1.55 \mathrm{E}-01$ & $-3.68 \mathrm{E}-01$ & $3.13 \mathrm{E}-01$ \\
$a_{5}$ & $2.64 \mathrm{E}-02$ & $-1.26 \mathrm{E}-01$ & $1.66 \mathrm{E}-01$ & $1.18 \mathrm{E}-01$ & $-4.11 \mathrm{E}-01$ \\
$a_{6}$ & $-1.86 \mathrm{E}-02$ & $9.13 \mathrm{E}-02$ & $-1.48 \mathrm{E}-01$ & $1.49 \mathrm{E}-03$ & $3.00 \mathrm{E}-01$ \\
$a_{7}$ & $1.38 \mathrm{E}-02$ & $-6.93 \mathrm{E}-02$ & $1.27 \mathrm{E}-01$ & $-5.57 \mathrm{E}-02$ & $-1.83 \mathrm{E}-01$ \\
$a_{8}$ & $-1.07 \mathrm{E}-02$ & $5.44 \mathrm{E}-02$ & $-1.07 \mathrm{E}-01$ & $7.83 \mathrm{E}-02$ & $9.63 \mathrm{E}-02$ \\
$a_{9}$ & $8.56 \mathrm{E}-03$ & $-4.38 \mathrm{E}-02$ & $9.14 \mathrm{E}-02$ & $-8.56 \mathrm{E}-02$ & $-3.76 \mathrm{E}-02$ \\
$a_{10}$ & $-7.01 \mathrm{E}-03$ & $3.61 \mathrm{E}-02$ & $-7.83 \mathrm{E}-02$ & $8.55 \mathrm{E}-02$ & $-4.48 \mathrm{E}-04$ \\
\hline
\end{tabular}

TABLE 1. Eigenvalues for the antisymmetric eigenfunctions after a $100 \times 100$ approximation, the free-end case. Eigenfunctions are such that $\sum a_{i}^{2}=1$.

with $c_{0 n}, n \geq 1$, and left hand side by a zeros-row. This is true since by (35) and (28),

$$
\begin{aligned}
\sum_{n \geq 1} c_{0 n} \pi\left(n-\frac{1}{2}\right) a_{n} & =\frac{1}{\pi} \int_{-1}^{1} \frac{\sum_{n} a_{n}\left(n-\frac{1}{2}\right) \pi \cos \left(\left(n-\frac{1}{2}\right) \pi x^{\prime}\right)}{\sqrt{1-x^{\prime 2}}} d x^{\prime} \\
& =\frac{1}{\pi} \int_{-1}^{1} \frac{S^{\prime}\left(x^{\prime}\right)}{\sqrt{1-x^{\prime 2}}} d x^{\prime} \\
& =0 .
\end{aligned}
$$

Therefore we can write system (40) in the following way:

$$
-\lambda F^{T} \overline{D E} \mathbf{a}=\pi\left[\left(i-\frac{1}{2}\right) \delta_{i j}\right] \mathbf{a}
$$

where $F$ is given by (41) and $\overline{D E}$ denotes matrix $D E$ with zeros in the first row. In Table 1 we find the first eigenvalues and the corresponding eigenvectors by an $N \times N$ approximation of the matrices. Moreover we observe the convergence to the limiting eigenvalue $\lambda_{1}$, see Figure 2 . The obtained values are consistent with those obtained in [11] by means of full numerical simulation and older approaches in [6] and [13], improving them by providing better accuracy.

Hence, by (8) and (21), we can obtain solutions $\widetilde{\zeta}$ of the form

$$
\widetilde{\zeta}(t, x)=\sum_{n \geq 1}\left(\alpha_{n} \cos \left(\sqrt{\lambda_{n}} t\right)+\beta_{n} \sin \left(\sqrt{\lambda_{n}} t\right)\right) \sin \left(\left(n-\frac{1}{2}\right) \pi x^{\prime}\right),
$$

where $\alpha_{n}, \beta_{n}$ are constants and the $\lambda$ 's are the corresponding eigenvalues of antisymmetric solutions. 


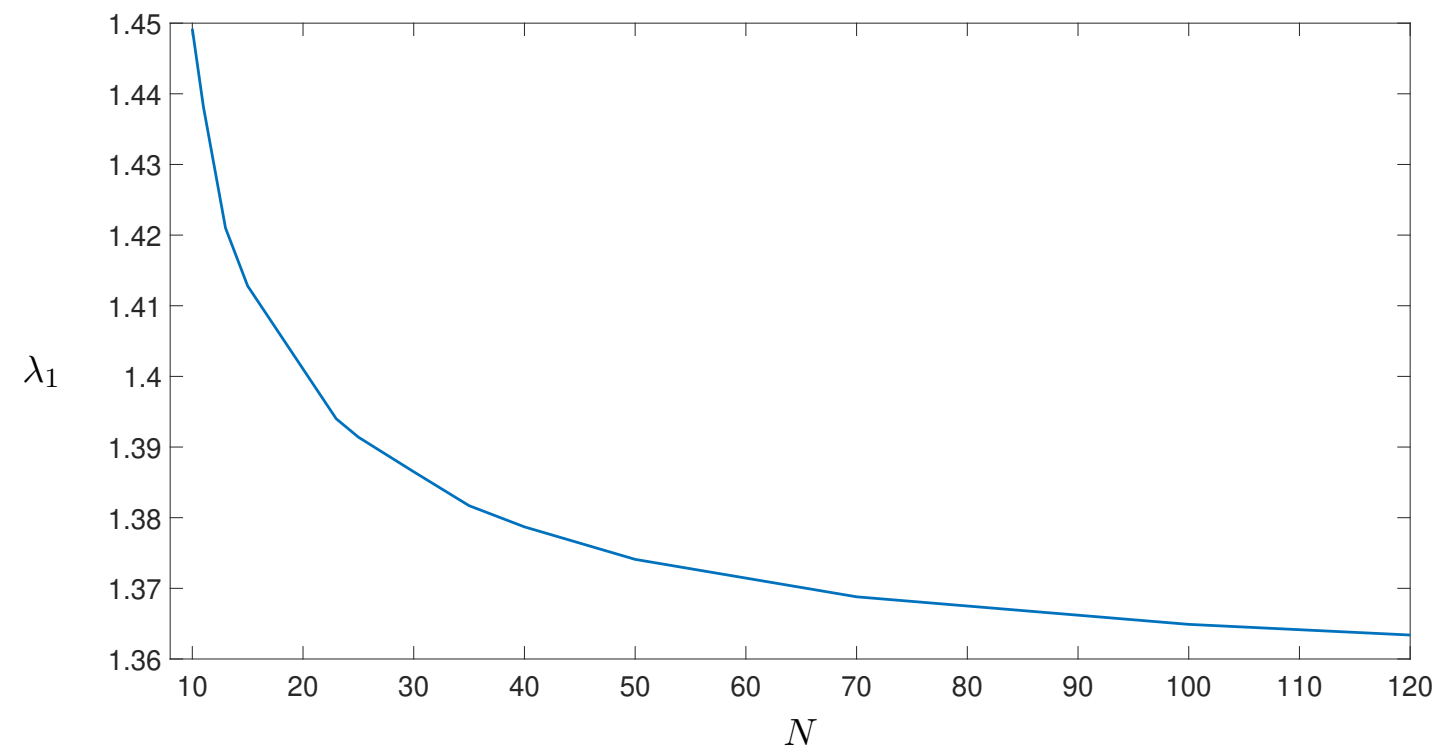

Figure 2. Error as a function of matrix size $N$.

3.2. Symmetric solution with respect to $x^{\prime}=0$, the free-end case. We consider now series of the cosine functions, corresponding to the symmetric case with respect to the origin in $[-1,1]$. Let $S\left(x^{\prime}\right)=\sum_{n \geq 1} b_{n} \cos \left(n \pi x^{\prime}\right)$, and the Tchebyshev polynomial expansions:

$$
\begin{aligned}
& \sin \left(n \pi x^{\prime}\right)=\sum_{k \geq 1} c_{k n} T_{k}\left(x^{\prime}\right), \\
& \cos \left(n \pi x^{\prime}\right)=\sum_{r \geq 1} e_{r n} \sqrt{1-x^{\prime 2}} U_{r-1}\left(x^{\prime}\right),
\end{aligned}
$$

in such a way that

$$
S\left(x^{\prime}\right)=\sum_{n, r} e_{r n} b_{n} \sqrt{1-x^{\prime 2}} U_{r-1}\left(x^{\prime}\right)
$$

By the orthogonality of the Tchebyshev polynomials,

$$
\begin{aligned}
& c_{k n}=\frac{2}{\pi} \int_{-1}^{1} \sin \left(n \pi x^{\prime}\right) \frac{T_{k}\left(x^{\prime}\right)}{\sqrt{1-x^{\prime 2}}} d x^{\prime}, \\
& e_{r n}=\frac{2}{\pi} \int_{-1}^{1} \cos \left(n \pi x^{\prime}\right) U_{r-1}\left(x^{\prime}\right) d x^{\prime} .
\end{aligned}
$$


Therefore, replacing (46) into (31)

$$
\begin{aligned}
\lambda & \sum_{n, r} e_{r n} b_{n} \sqrt{1-x^{\prime 2}} U_{r-1}\left(x^{\prime}\right) \\
& =-\frac{1}{\pi}\left(1-x^{\prime 2}\right)\left(1+\sqrt{1-x^{\prime 2}}\right) P . V \int_{-1}^{1} \frac{\sum_{n} n \pi b_{n} \sin (n \pi \zeta)}{\sqrt{1-\zeta^{2}}\left(x^{\prime}-\zeta\right)} d \zeta \\
& =-\frac{1}{\pi}\left(1-x^{\prime 2}\right)\left(1+\sqrt{1-x^{\prime 2}}\right) P . V \int_{-1}^{1} \frac{\sum_{n, k} n \pi c_{k n} b_{n} T_{k}(\zeta)}{\sqrt{1-\zeta^{2}}\left(x^{\prime}-\zeta\right)} d \zeta \\
& =-\left(1-x^{\prime 2}\right)\left(1+\sqrt{1-x^{\prime 2}}\right) \sum_{n, k} n \pi c_{k n} b_{n}\left(\frac{1}{\pi} P . V \int_{-1}^{1} \frac{T_{k}(\zeta)}{\sqrt{1-\zeta^{2}}\left(x^{\prime}-\zeta\right)} d \zeta\right) \\
& =\left(1-x^{\prime 2}\right)\left(1+\sqrt{1-x^{\prime 2}}\right) \sum_{n, k} n \pi c_{k n} b_{n} U_{k-1}\left(x^{\prime}\right) .
\end{aligned}
$$

Namely, for all $n, r, k \geq 1$

$$
\lambda \sum_{n, r} e_{r n} b_{n} \frac{U_{r-1}\left(x^{\prime}\right)}{\sqrt{1-x^{\prime 2}}\left(1+\sqrt{1-x^{\prime 2}}\right)}=\sum_{n, k} n \pi c_{k n} b_{n} U_{k-1}\left(x^{\prime}\right) .
$$

Then, if we write

$$
\frac{U_{r-1}\left(x^{\prime}\right)}{\sqrt{1-x^{\prime 2}}\left(1+\sqrt{1-x^{\prime 2}}\right)}=\sum_{k} d_{k r} U_{k-1}\left(x^{\prime}\right)
$$

for some $d_{k r}$ and we replace in (49):

$$
\lambda \sum_{n, k, r} d_{k r} e_{r n} b_{n} U_{k-1}\left(x^{\prime}\right)=\sum_{n, k} n \pi c_{k n} b_{n} U_{k-1}\left(x^{\prime}\right) .
$$

Thus we get the eigenvalue problem

$$
\lambda \sum_{n, r} d_{k r} e_{r n} b_{n}=\sum_{n} n \pi c_{k n} b_{n}
$$

with $c_{k n}, e_{r n}, d_{k r}$ as in (47), (48), (38) respectively and $n, r, k \geq 1$. In matrix form, (51) can be written as

$$
\lambda D E \mathbf{b}=\pi C\left[i \delta_{i j}\right] \mathbf{b},
$$

with $D=\left(d_{k r}\right), E=\left(e_{r n}\right), C=\left(c_{k n}\right)$ and $\mathbf{b}=\left(b_{n}\right)^{T}$.

Let

$$
f_{k n}=\int_{-1}^{1} \sin \left(n \pi x^{\prime}\right) T_{k}\left(x^{\prime}\right) d x^{\prime}, \quad k \geq 1, n \geq 1
$$




\begin{tabular}{cccccr}
\hline- & $\lambda_{1}=2.695$ & $\lambda_{2}=6.296$ & $\lambda_{3}=9.5$ & $\lambda_{4}=12.741$ & $\lambda_{5}=16.001$ \\
\hline$b_{1}$ & $9.14 \mathrm{E}-01$ & $8.76 \mathrm{E}-01$ & $3.07 \mathrm{E}-01$ & $1.01 \mathrm{E}-01$ & $6.95 \mathrm{E}-03$ \\
$b_{2}$ & $-3.32 \mathrm{E}-01$ & $2.61 \mathrm{E}-01$ & $8.92 \mathrm{E}-01$ & $6.44 \mathrm{E}-01$ & $3.04 \mathrm{E}-01$ \\
$b_{3}$ & $1.74 \mathrm{E}-01$ & $-2.58 \mathrm{E}-01$ & $-2.13 \mathrm{E}-01$ & $5.65 \mathrm{E}-01$ & $7.80 \mathrm{E}-01$ \\
$b_{4}$ & $-1.09 \mathrm{E}-01$ & $1.99 \mathrm{E}-01$ & $-2.41 \mathrm{E}-02$ & $-4.32 \mathrm{E}-01$ & $7.62 \mathrm{E}-02$ \\
$b_{5}$ & $7.44 \mathrm{E}-02$ & $-1.52 \mathrm{E}-01$ & $9.84 \mathrm{E}-02$ & $2.29 \mathrm{E}-01$ & $-3.24 \mathrm{E}-01$ \\
$b_{6}$ & $-5.44 \mathrm{E}-02$ & $1.18 \mathrm{E}-01$ & $-1.17 \mathrm{E}-01$ & $-9.40 \mathrm{E}-02$ & $3.12 \mathrm{E}-01$ \\
$b_{7}$ & $4.16 \mathrm{E}-02$ & $-9.42 \mathrm{E}-02$ & $1.15 \mathrm{E}-01$ & $1.52 \mathrm{E}-02$ & $-2.35 \mathrm{E}-01$ \\
$b_{8}$ & $-3.28 \mathrm{E}-02$ & $7.66 \mathrm{E}-02$ & $-1.06 \mathrm{E}-01$ & $2.87 \mathrm{E}-02$ & $1.57 \mathrm{E}-01$ \\
$b_{9}$ & $2.66 \mathrm{E}-02$ & $-6.34 \mathrm{E}-02$ & $9.59 \mathrm{E}-02$ & $-5.21 \mathrm{E}-02$ & $-9.38 \mathrm{E}-02$ \\
$b_{10}$ & $-2.21 \mathrm{E}-02$ & $5.33 \mathrm{E}-02$ & $-8.58 \mathrm{E}-02$ & $6.37 \mathrm{E}-02$ & $4.73 \mathrm{E}-02$ \\
\hline
\end{tabular}

TABLE 2. Eigenvalues for the symmetric eigenfunctions after a $100 \times 100$ approximation, the free-end case. Eigenfunctions are such that $\sum b_{i}^{2}=1$.

and assume $\sin \left(n \pi x^{\prime}\right)=\sum_{k} \alpha_{k n} \frac{T_{k}\left(x^{\prime}\right)}{\sqrt{1-x^{\prime 2}}}$. By the orthogonality of the Tchebyshev polynomials,

$$
\begin{aligned}
\alpha_{k n} & =\frac{2}{\pi} \int_{-1}^{1} \sin \left(n \pi x^{\prime}\right) T_{k}\left(x^{\prime}\right) d x^{\prime}, \\
& =\frac{2}{\pi} f_{k n} .
\end{aligned}
$$

Therefore, by (44)

$$
\begin{aligned}
\delta_{m n} & =\int_{-1}^{1} \sin \left(n \pi x^{\prime}\right) \sin \left(m \pi x^{\prime}\right) d x^{\prime} \\
& =\int_{-1}^{1} \sum_{k} c_{k n} T_{k}\left(x^{\prime}\right) \sum_{k^{\prime}} \alpha_{k^{\prime} m} \frac{T_{k^{\prime}}\left(x^{\prime}\right)}{\sqrt{1-x^{\prime 2}}} d x^{\prime} \\
& =\frac{2}{\pi} \int_{-1}^{1} \sum_{k} c_{k n} T_{k}\left(x^{\prime}\right) \sum_{k^{\prime}} f_{k^{\prime} m} \frac{T_{k^{\prime}}\left(x^{\prime}\right)}{\sqrt{1-x^{\prime 2}}} d x^{\prime} \\
& =\sum_{k, k^{\prime}} f_{k^{\prime} m} c_{k n} \frac{2}{\pi} \int_{-1}^{1} \frac{T_{k}\left(x^{\prime}\right) T_{k^{\prime}}\left(x^{\prime}\right)}{\sqrt{1-x^{\prime 2}}} d x^{\prime} \\
& =\sum_{k \geq 1} f_{k m} c_{k n} .
\end{aligned}
$$

Namely,

$$
F^{T} C=I
$$

with $F=\left(f_{k n}\right)$ given by $(53)$.

Note that in this case we don't need to modify system (52) in its first row, since condition $(28)$ is automatically satisfied by the symmetry of $S(x)$. 
Therefore, system (52) can be written as

$$
\lambda F^{T} D E \mathbf{b}=\pi\left[i \delta_{i j}\right] \mathbf{b},
$$

where $F$ is given by (53). In Table 2 we find the first eigenvalues and the corresponding eigenvectors by a $100 \times 100$ approximation of the matrices.

Notice that in the two cases above, at the contact line, we are not including some other physical phenomena like viscosity, contact angle hysteresis, pressure, etc. Since we are working in gravity waves, we expect the frequencies are not influenced by the dynamics near the contact lines.

\subsection{Antisymmetric solution with respect to $x^{\prime}=0$, the pinned-end}

case. Proceeding similarly as the two cases above, let $S\left(x^{\prime}\right)=\sum_{n \geq 1} \widetilde{a}_{n} \sin \left(n \pi x^{\prime}\right)$ and the Tchebyshev polynomial expansions:

$$
\begin{aligned}
\cos \left(n \pi x^{\prime}\right) & =\sum_{k \geq 0} c_{k n} T_{k}\left(x^{\prime}\right) \\
\sin \left(n \pi x^{\prime}\right) & =\sum_{r \geq 1} e_{r n} \sqrt{1-x^{\prime 2}} U_{r-1}\left(x^{\prime}\right),
\end{aligned}
$$

in such a way that

$$
S\left(x^{\prime}\right)=\sum_{n, r} e_{r n} \widetilde{a}_{n} \sqrt{1-x^{\prime 2}} U_{r-1}\left(x^{\prime}\right) .
$$

By the orthogonality of the Tchebyshev polynomials,

$$
\begin{gathered}
c_{k n}= \begin{cases}\frac{2}{\pi} \int_{-1}^{1} \cos \left(n \pi x^{\prime}\right) \frac{T_{k}\left(x^{\prime}\right)}{\sqrt{1-x^{\prime 2}}} d x^{\prime}, & k \geq 1, \\
\frac{1}{\pi} \int_{-1}^{1} \cos \left(n \pi x^{\prime}\right) \frac{1}{\sqrt{1-x^{\prime 2}}} d x^{\prime}, & k=0,\end{cases} \\
e_{r n}=\frac{2}{\pi} \int_{-1}^{1} \sin \left(n \pi x^{\prime}\right) U_{r-1}\left(x^{\prime}\right) d x^{\prime} .
\end{gathered}
$$

Therefore, replacing (56) into (31)

$\lambda \sum_{n, r} e_{r n} \widetilde{a}_{n} \sqrt{1-x^{\prime 2}} U_{r-1}\left(x^{\prime}\right)=-\left(1-x^{\prime 2}\right)\left(1+\sqrt{1-x^{\prime 2}}\right) \sum_{n, k} n \pi c_{k n} \widetilde{a}_{n} U_{k-1}\left(x^{\prime}\right)$.

Namely, for all $n, k, r \geq 1$

$$
-\lambda \sum_{n, r} e_{r n} \widetilde{a}_{n} \frac{U_{r-1}\left(x^{\prime}\right)}{\sqrt{1-x^{\prime 2}}\left(1+\sqrt{1-x^{\prime 2}}\right)}=\sum_{n, k} n \pi c_{k n} \widetilde{a}_{n} U_{k-1}\left(x^{\prime}\right) .
$$

Then, by (38)

$$
-\lambda \sum_{n, k, r} d_{k r} e_{r n} \widetilde{a}_{n} U_{k-1}\left(x^{\prime}\right)=\sum_{n, k} n \pi c_{k n} \widetilde{a}_{n} U_{k-1}\left(x^{\prime}\right) .
$$

Thus we get the eigenvalue problem

$$
-\lambda \sum_{n, r} d_{k r} e_{r n} \widetilde{a}_{n}=\sum_{n} n \pi c_{k n} \widetilde{a}_{n}
$$


OSCILLATIONS AT THE BOUNDARY OF A ROUND SHAPED CONTAINER 18

\begin{tabular}{lccccr}
\hline- & $\lambda_{1}=5.7726$ & $\lambda_{2}=7.9596$ & $\lambda_{3}=11.1673$ & $\lambda_{4}=14.2409$ & $\lambda_{5}=17.334$ \\
\hline$\widetilde{a}_{1}$ & $9.61 \mathrm{E}-01$ & $6.42 \mathrm{E}-01$ & $8.44 \mathrm{E}-01$ & $7.37 \mathrm{E}-01$ & $7.55 \mathrm{E}-01$ \\
$\widetilde{a}_{2}$ & $-2.49 \mathrm{E}-01$ & $-6.35 \mathrm{E}-01$ & $1.37 \mathrm{E}-01$ & $-5.38 \mathrm{E}-01$ & $-1.78 \mathrm{E}-01$ \\
$\widetilde{a}_{3}$ & $1.03 \mathrm{E}-01$ & $3.41 \mathrm{E}-01$ & $2.57 \mathrm{E}-01$ & $-2.28 \mathrm{E}-01$ & $5.88 \mathrm{E}-01$ \\
$\widetilde{a}_{4}$ & $-5.29 \mathrm{E}-02$ & $-1.98 \mathrm{E}-01$ & $-2.76 \mathrm{E}-01$ & $2.60 \mathrm{E}-02$ & $7.29 \mathrm{E}-02$ \\
$\widetilde{a}_{5}$ & $3.10 \mathrm{E}-02$ & $1.24 \mathrm{E}-01$ & $2.27 \mathrm{E}-01$ & $1.06 \mathrm{E}-01$ & $-1.44 \mathrm{E}-01$ \\
$\widetilde{a}_{6}$ & $-1.98 \mathrm{E}-02$ & $-8.29 \mathrm{E}-02$ & $-1.77 \mathrm{E}-01$ & $-1.53 \mathrm{E}-01$ & $6.86 \mathrm{E}-02$ \\
$\widetilde{a}_{7}$ & $1.35 \mathrm{E}-02$ & $5.81 \mathrm{E}-02$ & $1.38 \mathrm{E}-01$ & $1.60 \mathrm{E}-01$ & $7.53 \mathrm{E}-03$ \\
$\widetilde{a}_{8}$ & $-9.60 \mathrm{E}-03$ & $-4.23 \mathrm{E}-02$ & $-1.08 \mathrm{E}-01$ & $-1.50 \mathrm{E}-01$ & $-5.82 \mathrm{E}-02$ \\
$\widetilde{a}_{9}$ & $7.10 \mathrm{E}-03$ & $3.18 \mathrm{E}-02$ & $8.54 \mathrm{E}-02$ & $1.34 \mathrm{E}-01$ & $8.64 \mathrm{E}-02$ \\
$\widetilde{a}_{10}$ & $-5.40 \mathrm{E}-03$ & $-2.45 \mathrm{E}-02$ & $-6.86 \mathrm{E}-02$ & $-1.17 \mathrm{E}-01$ & $-9.93 \mathrm{E}-02$ \\
\hline
\end{tabular}

TABle 3. Eigenvalues for the antisymmetric eigenfunctions after a $100 \times 100$ approximation, the pinned-end case. Eigenfunctions are such that $\sum \widetilde{a}_{i}^{2}=1$.

with $c_{k n}, e_{r n}$ as in (57), (58), respectively and $r, k, n \geq 1$. In matrix form, if we denote $\widetilde{\mathbf{a}}=\left(\widetilde{a}_{n}\right)^{T}$, (60) can be written as

$$
-\lambda D E \widetilde{\mathbf{a}}=\pi C\left[i \delta_{i j}\right] \widetilde{\mathbf{a}} .
$$

As we did before, defining $F=\left(f_{k n}\right)$,

$$
f_{k n}=\int_{-1}^{1} \cos \left(n \pi x^{\prime}\right) T_{k}\left(x^{\prime}\right) d x^{\prime}, \quad k \geq 0, n \geq 1
$$

we have

$$
F^{T} C=I
$$

and (61) is equivalent to

$$
-\lambda F^{T} \overline{D E} \widetilde{\mathbf{a}}=\pi\left[i \delta_{i j}\right] \widetilde{\mathbf{a}},
$$

with $F$ and $E$ given by (62) and (58) respectively. Note that (64) is true as long as (see condition (28))

$$
\begin{aligned}
0 & =\int_{-1}^{1} \frac{S^{\prime}(x)}{\sqrt{1-x^{2}}} d x=\sum_{n} c_{0 n} n \pi \widetilde{a}_{n} \\
& =\sum_{n} n \widetilde{a}_{n} \int_{-1}^{1} \frac{\cos \left(n \pi x^{\prime}\right)}{\sqrt{1-x^{\prime 2}}} d x^{\prime} \\
& =\pi \sum_{n} n \widetilde{a}_{n} J_{0}(\pi n) .
\end{aligned}
$$


3.4. Symmetric solution with respect to $x^{\prime}=0$, the pinned-end case (mass conservation extra condition). Finally, Let $S\left(x^{\prime}\right)=\sum_{n \geq 1} \widetilde{b}_{n} \cos \left(\left(n-\frac{1}{2}\right) \pi x^{\prime}\right)$. In this case we have to add the extra condition (mass conservation):

$$
\int_{-1}^{1} \phi(x, 0, t) d x=0
$$

By (30)

$$
\begin{aligned}
\int_{-1}^{1} \phi(x, 0, t) d x & =\int_{-1}^{1} \frac{\widetilde{\phi}\left(x^{\prime}, 0, t\right)}{\sqrt{1-x^{\prime 2}}\left(1+\sqrt{1-x^{\prime 2}}\right)} d x^{\prime} \\
& =\int_{-1}^{1} \frac{A(t) S\left(x^{\prime}\right)}{\sqrt{1-x^{\prime 2}}\left(1+\sqrt{1-x^{\prime 2}}\right)} d x^{\prime}
\end{aligned}
$$

Then, replacing $S$ and using (47)

$$
0=\sum_{n} \widetilde{b}_{n} \int_{-1}^{1} \frac{\cos \left(\left(n-\frac{1}{2}\right) \pi x^{\prime}\right)}{\sqrt{1-x^{\prime 2}}\left(1+\sqrt{1-x^{\prime 2}}\right)} d x^{\prime}=\sum_{n} \widetilde{b}_{n} \omega_{n},
$$

where

$$
\omega_{n}=\int_{-1}^{1} \frac{\cos \left(\left(n-\frac{1}{2}\right) \pi x^{\prime}\right)}{\sqrt{1-x^{\prime 2}}\left(1+\sqrt{1-x^{\prime 2}}\right)} d x^{\prime} .
$$

Proceeding as in the symmetric (free end) case, let

$$
\begin{aligned}
& \cos \left(\left(n-\frac{1}{2}\right) \pi x^{\prime}\right)=\sum_{r \geq 1} e_{r n} \sqrt{1-x^{\prime 2}} U_{r-1}\left(x^{\prime}\right), \\
& \sin \left(\left(n-\frac{1}{2}\right) \pi x^{\prime}\right)=\sum_{k \geq 1} c_{k n} T_{k}\left(x^{\prime}\right),
\end{aligned}
$$

where

$$
\begin{aligned}
& e_{r n}=\frac{2}{\pi} \int_{-1}^{1} \cos \left(\left(n-\frac{1}{2}\right) \pi x^{\prime}\right) U_{r-1}\left(x^{\prime}\right) d x^{\prime}, \\
& c_{k n}=\frac{2}{\pi} \int_{-1}^{1} \sin \left(\left(n-\frac{1}{2}\right) \pi x^{\prime}\right) \frac{T_{k}\left(x^{\prime}\right)}{\sqrt{1-x^{\prime 2}}} d x^{\prime} .
\end{aligned}
$$

By replacing $S\left(x^{\prime}\right)$ into $(31)$,

$$
\begin{aligned}
& \lambda \sum_{n, r} e_{r n} \widetilde{b}_{n} \sqrt{1-x^{\prime 2}} U_{r-1}\left(x^{\prime}\right) \\
= & \left(1-x^{\prime 2}\right)\left(1+\sqrt{1-x^{\prime 2}}\right) \sum_{n, k}\left(n-\frac{1}{2}\right) \pi c_{k n} \widetilde{b}_{n} U_{k-1}\left(x^{\prime}\right) .
\end{aligned}
$$

Namely, for all $n, r, k \geq 1$,

$$
\lambda \sum_{n, r} e_{r n} \widetilde{b}_{n} \frac{U_{r-1}\left(x^{\prime}\right)}{\sqrt{1-x^{\prime 2}}\left(1+\sqrt{1-x^{\prime 2}}\right)}=\sum_{n, k}\left(n-\frac{1}{2}\right) \pi c_{k n} \widetilde{b}_{n} U_{k-1}\left(x^{\prime}\right) .
$$




\begin{tabular}{cccccr}
\hline- & $\lambda_{1}=0.736$ & $\lambda_{2}=4.645$ & $\lambda_{3}=8.173$ & $\lambda_{4}=11.63$ & $\lambda_{5}=15.08$ \\
\hline$\widetilde{b}_{1}$ & $9.54 \mathrm{E}-01$ & $6.75 \mathrm{E}-01$ & $1.31 \mathrm{E}-01$ & $2.90 \mathrm{E}-02$ & $-2.71 \mathrm{E}-03$ \\
$\widetilde{b}_{2}$ & $-2.45 \mathrm{E}-01$ & $5.67 \mathrm{E}-01$ & $8.84 \mathrm{E}-01$ & $4.36 \mathrm{E}-01$ & $1.42 \mathrm{E}-01$ \\
$\widetilde{b}_{3}$ & $1.27 \mathrm{E}-01$ & $-3.41 \mathrm{E}-01$ & $1.03 \mathrm{E}-01$ & $8.07 \mathrm{E}-01$ & $7.03 \mathrm{E}-01$ \\
$\widetilde{b}_{4}$ & $-8.08 \mathrm{E}-02$ & $2.22 \mathrm{E}-01$ & $-2.29 \mathrm{E}-01$ & $-2.81 \mathrm{E}-01$ & $4.69 \mathrm{E}-01$ \\
$\widetilde{b}_{5}$ & $5.73 \mathrm{E}-02$ & $-1.56 \mathrm{E}-01$ & $2.17 \mathrm{E}-01$ & $1.03 \mathrm{E}-02$ & $-4.35 \mathrm{E}-01$ \\
$\widetilde{b}_{6}$ & $-4.33 \mathrm{E}-02$ & $1.16 \mathrm{E}-01$ & $-1.83 \mathrm{E}-01$ & $9.73 \mathrm{E}-02$ & $2.39 \mathrm{E}-01$ \\
$\widetilde{b}_{7}$ & $3.41 \mathrm{E}-02$ & $-8.95 \mathrm{E}-02$ & $1.51 \mathrm{E}-01$ & $-1.33 \mathrm{E}-01$ & $-8.73 \mathrm{E}-02$ \\
$\widetilde{b}_{8}$ & $-2.77 \mathrm{E}-02$ & $7.13 \mathrm{E}-02$ & $-1.25 \mathrm{E}-01$ & $1.38 \mathrm{E}-01$ & $-6.05 \mathrm{E}-03$ \\
$\widetilde{b}_{9}$ & $2.29 \mathrm{E}-02$ & $-5.81 \mathrm{E}-02$ & $1.04 \mathrm{E}-01$ & $-1.32 \mathrm{E}-01$ & $5.84 \mathrm{E}-02$ \\
$\widetilde{b}_{10}$ & $-1.94 \mathrm{E}-02$ & $4.83 \mathrm{E}-02$ & $-8.77 \mathrm{E}-02$ & $1.20 \mathrm{E}-01$ & $-8.52 \mathrm{E}-02$ \\
\hline
\end{tabular}

TABLE 4. Eigenvalues for the symmetric eigenfunctions after a $100 \times 100$ approximation, the pinned-end case. Eigenfunctions are such that $\sum \widetilde{b}_{i}^{2}=1$.

Then, by (38)

$$
\lambda \sum_{n, k, r} d_{k r} e_{r n} \widetilde{b}_{n} U_{k-1}\left(x^{\prime}\right)=\sum_{n, k}\left(n-\frac{1}{2}\right) \pi c_{k n} \widetilde{b}_{n} U_{k-1}\left(x^{\prime}\right) .
$$

Thus we get the eigenvalue problem

$$
\lambda \sum_{n, r} d_{k r} e_{r n} \widetilde{b}_{n}=\sum_{n}\left(n-\frac{1}{2}\right) \pi c_{k n} \widetilde{b}_{n}
$$

with $e_{r n}, c_{k n}$ as in (67), (68), respectively and $n, r, k \geq 1$. In matrix form, by denoting $\widetilde{\mathbf{b}}=\left(\widetilde{b}_{n}\right)^{T}$, (69) can be written as

$$
\lambda D E \widetilde{\mathbf{b}}=\pi C\left[\left(i-\frac{1}{2}\right) \delta_{i j}\right] \widetilde{\mathbf{b}} .
$$

As we did before, defining $F=\left(f_{k n}\right)$,

$$
f_{k n}=\int_{-1}^{1} \sin \left(\left(n-\frac{1}{2}\right) \pi x^{\prime}\right) T_{k}\left(x^{\prime}\right) d x^{\prime}, \quad k \geq 1, n \geq 1,
$$

we have

$$
F^{T} C=I
$$

and (70) is equivalent to

$$
\lambda F^{T} D E \widetilde{\mathbf{b}}=\pi\left[\left(i-\frac{1}{2}\right) \delta_{i j}\right] \widetilde{\mathbf{b}},
$$

with $F$ and $E$ given by (71) and (67) respectively. Finally, we have to add the extra mass conservation condition (65) in the first row of system (72), by adding a zero row to $F^{T} D E$ and $\omega$ as the first row of diagonal matrix 

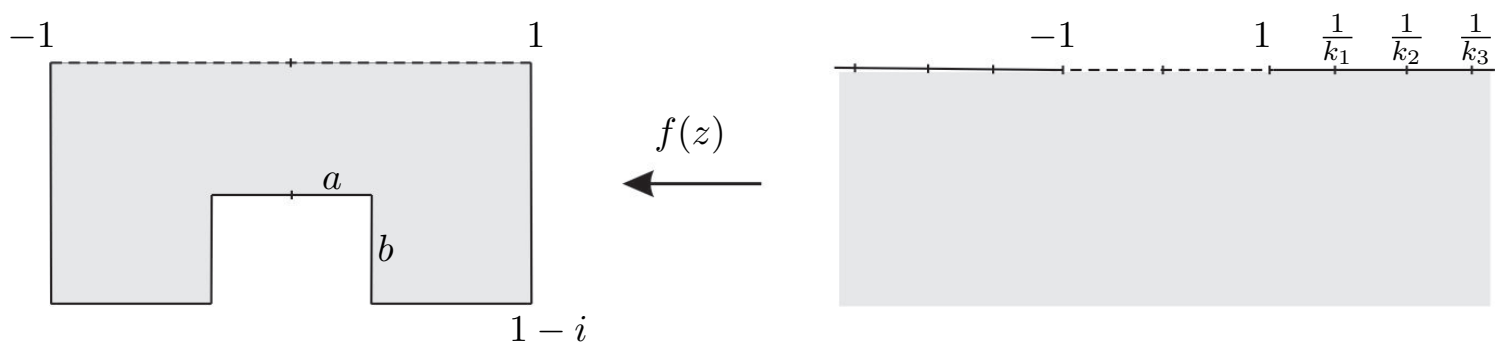

FiguRe 3. Geometry of the problem for a polygonal container mapped into the half-plane by the Schwarz-Christoffel $\operatorname{map} f$.

$\left[\left(i-\frac{1}{2}\right) \delta_{i j}\right]$, where $\omega$ is given by (66). In Table 4 we present some frequencies and the corresponding coefficients after a $100 \times 100$ approximation.

\section{WAVES IN A CHANNEL With A RECTANGUlaR UNDERWATER OBSTACLE}

In this section, we apply the general representation for the evolution of the free surface as an integrodifferential equation, obtained in (24), to study a different type of geometry. Namely, we want to compute the frequencies for the oscillations of the free boundary, of a polygonal domain by using the Schwarz-Christoffel map (see [21]). This problem has practical interests in oceanography in relation to the design of uneven bottoms transforming prescribed frequencies into decreasing ones [7, 16, 18].

Let $0<a, b<1$. The Schwarz-Christoffel map $f(z)$, given by

$$
f(z)=\alpha \int_{0}^{z} \frac{\sqrt{1-k_{3}^{2} \zeta^{2}}}{\sqrt{1-\zeta^{2}} \sqrt{1-k_{1}^{2} \zeta^{2}} \sqrt{1-k_{2}^{2} \zeta^{2}}} d \zeta+\beta,
$$

with $\alpha$ and $\beta$ two constants, is the conformal mapping of the half-plane $\operatorname{Im}\{w\}<0$ onto the 8-gon of Figure 3 . Note that $\pm 1, \pm 1 / k_{1}, \pm 1 / k_{2}, \pm 1 / k_{3}$ are mapped into the 8-gon vertex, symmetrically and $0<k_{1}, k_{2}, k_{3}<1$.

Since $f(0)=0$ we have $\beta=0$. Moreover the relations between $k_{1}, k_{2}$ and $k_{3}$ with $a$ and $b$, can be determined in the following way: let

$$
g(\zeta)=\frac{\sqrt{1-k_{3}^{2} \zeta^{2}}}{\sqrt{1-\zeta^{2}} \sqrt{1-k_{1}^{2} \zeta^{2}} \sqrt{1-k_{2}^{2} \zeta^{2}}} .
$$

Since $f(1)=1$,

$$
1=\alpha \int_{0}^{1} g(\zeta) d \zeta
$$

By the condition $f\left(1 / k_{1}\right)=1-i$, we have

$$
1-i=\alpha \int_{0}^{1 / k_{1}} g(\zeta) d \zeta,
$$


and therefore, using (73)

$$
\begin{aligned}
-i & =\alpha \int_{0}^{1 / k_{1}} g(\zeta) d \zeta-1 \\
& =\alpha \int_{0}^{1 / k_{1}} g(\zeta) d \zeta-\alpha \int_{0}^{1} g(\zeta) d \zeta \\
& =\alpha \int_{1}^{1 / k_{1}} g(\zeta) d \zeta .
\end{aligned}
$$

In the same way, $f\left(1 / k_{2}\right)=1-a-i$ implies

$$
1-a-i=\alpha \int_{0}^{1 / k_{2}} g(\zeta) d \zeta
$$

and then, by (73), (74)

$$
\begin{aligned}
-a & =\alpha \int_{0}^{1 / k_{2}} g(\zeta) d \zeta-1+i \\
& =\alpha \int_{0}^{1 / k_{2}} g(\zeta) d \zeta-\alpha \int_{0}^{1} g(\zeta) d \zeta-\alpha \int_{1}^{1 / k_{1}} g(\zeta) d \zeta \\
& =\alpha \int_{1 / k_{1}}^{1 / k_{2}} g(\zeta) d \zeta
\end{aligned}
$$

Finally, by the condition $f\left(1 / k_{3}\right)=1-a-i+b i$

$$
1-a-i+b i=\alpha \int_{0}^{1 / k_{3}} g(\zeta) d \zeta
$$

which implies, using (73), (74) and (75),

$$
\begin{aligned}
b i & =\alpha \int_{0}^{1 / k_{3}} g(\zeta) d \zeta-1+a+i \\
& =\alpha \int_{0}^{1 / k_{3}} g(\zeta) d \zeta-\alpha \int_{0}^{1} g(\zeta) d \zeta-\alpha \int_{1 / k_{1}}^{1 / k_{2}} g(\zeta) d \zeta-\alpha \int_{1}^{1 / k_{1}} g(\zeta) d \zeta \\
& =\alpha \int_{1 / k_{2}}^{1 / k_{3}} g(\zeta) d \zeta
\end{aligned}
$$

Putting together (73)-(76) we get the relations between $k_{1}, k_{2}, k_{3}$ and $a$, $b$, given by

$$
\begin{gathered}
1=\alpha \int_{0}^{1} g(\zeta) d \zeta, \quad-i=\frac{\int_{1}^{1 / k_{1}} g(\zeta) d \zeta}{\int_{0}^{1} g(\zeta) d \zeta} \\
-a=\frac{\int_{1 / k_{1}}^{1 / k_{2}} g(\zeta) d \zeta}{\int_{0}^{1} g(\zeta) d \zeta}, \quad b i=\frac{\int_{1 / k_{2}}^{1 / k_{3}} g(\zeta) d \zeta}{\int_{0}^{1} g(\zeta) d \zeta} .
\end{gathered}
$$

Equations (77), (78) allow to compute, numerically, the coefficients $k_{1}, k_{2}, k_{3}$ once $a, b$ are fixed (see Table 5 ), and therefore, it is possible to approximate 


\begin{tabular}{cccr}
\hline$(a, b) / k ' s$ & $k_{1}$ & $k_{2}$ & $k_{3}$ \\
\hline$(0,0)$ & 0.7071 & 0.7071 & 0.7071 \\
$(0.10,0.10)$ & 0.7797 & 0.7795 & 0.7772 \\
$(0.25,0.10)$ & 0.77288 & 0.7634 & 0.75244 \\
$(0.25,0.25)$ & 0.8739 & 0.8727 & 0.8603 \\
$(0.50,0.10)$ & 0.7556 & 0.6762 & 0.6432 \\
$(0.50,0.25)$ & 0.84465 & 0.81758 & 0.76511 \\
$(0.50,0.50)$ & 0.9658 & 0.9633 & 0.9304 \\
$(0.75,0.10)$ & 0.7365 & 0.48396 & 0.4202 \\
$(0.75,0.25)$ & 0.80384 & 0.6726 & 0.54066 \\
$(0.75,0.50)$ & 0.92968 & 0.89989 & 0.74873 \\
$(0.75,0.75)$ & 0.996399 & 0.995244 & 0.95654 \\
\hline
\end{tabular}

TABlE 5. Different values of $k_{1}, k_{2}, k_{3}$ corresponding to $a, b$ fixed.

the eigenvalue problem by an infinite matrix whose entries involve the coefficients $k_{1}, k_{2}, k_{3}$ and the Tchebyshev polynomials, as we did before.

Remark 1. In the particular case of the rectangle; that is, $a=0, b=0$, formulas above become

$$
f(z)=\alpha \int_{0}^{z} \frac{1}{\sqrt{1-\zeta^{2}} \sqrt{1-\kappa^{2} \zeta^{2}}} d \zeta
$$

where

$$
1=\alpha \int_{0}^{1} \frac{1}{\sqrt{1-\zeta^{2}} \sqrt{1-\kappa^{2} \zeta^{2}}} d \zeta, \quad-i=\alpha \int_{1}^{1 / \kappa} \frac{1}{\sqrt{1-\zeta^{2}} \sqrt{1-\kappa^{2} \zeta^{2}}} d \zeta .
$$

4.1. Antisymmetric solution with respect to $x^{\prime}=0$, the free-end case. Once we know the coefficients $k_{1}, k_{2}, k_{3}$, it is possible to compute explicitly the derivative of the conformal mapping $f$ and then, by (24), an explicit representation of the eigenvalues equation in terms of the Tchebyshev polynomials is obtained as follow:

$$
\begin{aligned}
\lambda S\left(x^{\prime}\right) & =\frac{1}{\pi} \sqrt{1-x^{\prime 2}} \frac{1}{\left|f^{\prime}(x)\right|} P . V \int_{-1}^{1} \frac{1}{\sqrt{1-\zeta^{2}}} \frac{S^{\prime}(\zeta)}{x^{\prime}-\zeta} d \zeta \\
& =\frac{1}{\pi}\left(1-x^{\prime 2}\right) \frac{\sqrt{1-k_{1}^{2} x^{\prime 2}} \sqrt{1-k_{2}^{2} x^{\prime 2}}}{\sqrt{1-k_{3}^{2} x^{\prime 2}}} P . V \int_{-1}^{1} \frac{1}{\sqrt{1-\zeta^{2}}} \frac{S^{\prime}(\zeta)}{x^{\prime}-\zeta} d \zeta .
\end{aligned}
$$

As we did before, for the antisymmetric solution with respect to $x^{\prime}=0$, the free-end case, if $S\left(x^{\prime}\right)=\sum_{n \geq 1} a_{n} \sin \left(\left(n-\frac{1}{2}\right) \pi x^{\prime}\right)$, by the equation above

$$
\lambda \sum_{n, r} e_{r n} a_{n} \sqrt{1-x^{\prime 2}} U_{r-1}\left(x^{\prime}\right)=-\left(1-x^{\prime 2}\right) \frac{\sqrt{1-k_{1}^{2} x^{\prime 2}} \sqrt{1-k_{2}^{2} x^{\prime 2}}}{\sqrt{1-k_{3}^{2} x^{\prime 2}}} \sum_{n, k}\left(n-\frac{1}{2}\right) \pi c_{k n} a_{n} U_{k-1}\left(x^{\prime}\right) .
$$




\begin{tabular}{cccccr}
\hline$(a, b) / \lambda$ & $\lambda_{1}$ & $\lambda_{2}$ & $\lambda_{3}$ & $\lambda_{4}$ & $\lambda_{5}$ \\
\hline$(0,0)$ & 0.78745 & 2.5799 & 4.3205 & 6.0881 & 7.8892 \\
$(0.10,0.10)$ & 0.72188 & 2.4409 & 4.0931 & 5.7756 & 7.4958 \\
$(0.25,0.10)$ & 0.72092 & 2.4388 & 4.0895 & 5.7706 & 7.4895 \\
$(0.25,0.25)$ & 0.59508 & 2.1631 & 3.6446 & 5.1659 & 6.7372 \\
$(0.50,0.10)$ & 0.72615 & 2.4491 & 4.1058 & 5.7925 & 7.5165 \\
$(0.50,0.25)$ & 0.60269 & 2.178 & 3.6653 & 5.1917 & 6.7665 \\
$(0.50,0.50)$ & 0.34452 & 1.5712 & 2.7441 & 4.0016 & 5.3542 \\
$(0.75,0.10)$ & 0.74262 & 2.4831 & 4.1611 & 5.8682 & 7.611 \\
$(0.75,0.25)$ & 0.63473 & 2.2439 & 3.7693 & 5.3302 & 6.9345 \\
$(0.75,0.50)$ & 0.37822 & 1.6277 & 2.7885 & 4.0204 & 5.3328 \\
$(0.75,0.75)$ & 0.12212 & 1.043 & 2.0888 & 3.2623 & 4.5574 \\
\hline
\end{tabular}

TABLE 6. First eigenvalues for different size of the bottom. Antisymmetric, free end case, taking $N=35$.

Namely

$-\lambda \sum_{n, r} e_{r n} a_{n} \frac{U_{r-1}\left(x^{\prime}\right)}{\sqrt{1-x^{\prime 2}}} \frac{\sqrt{1-k_{3}^{2} x^{\prime 2}}}{\sqrt{1-k_{1}^{2} x^{\prime 2}} \sqrt{1-k_{2}^{2} x^{\prime 2}}}=\sum_{n, k}\left(n-\frac{1}{2}\right) \pi c_{k n} a_{n} U_{k-1}\left(x^{\prime}\right)$.

Then, writing

$$
\frac{U_{r-1}\left(x^{\prime}\right)}{\sqrt{1-x^{\prime 2}}} \frac{\sqrt{1-k_{3}^{2} x^{\prime 2}}}{\sqrt{1-k_{1}^{2} x^{\prime 2}} \sqrt{1-k_{2}^{2} x^{\prime 2}}}=\sum_{k} d_{k r} U_{k-1}\left(x^{\prime}\right),
$$

(where we note that the matrix $D=\left(d_{k r}\right)$ contains the whole information on the container) the coefficients $d_{k r}$ are given by

$$
d_{k r}=\frac{2}{\pi} \int_{-1}^{1} U_{k-1}\left(x^{\prime}\right) U_{r-1}\left(x^{\prime}\right) \frac{\sqrt{1-k_{3}^{2} x^{\prime 2}}}{\sqrt{1-k_{1}^{2} x^{\prime 2}} \sqrt{1-k_{2}^{2} x^{\prime 2}}} d x^{\prime}
$$

and, for $\mathbf{a}=\left(a_{n}\right)^{T}$, the eigenvalues equation becomes

$$
-\lambda F^{T} \overline{D E} \mathbf{a}=\pi\left[\left(i-\frac{1}{2}\right) \delta_{i j}\right] \mathbf{a}
$$

with $E=\left(e_{r n}\right), F=\left(f_{k n}\right)$ given by $(36)$, (41) respectively and $D=\left(d_{k r}\right)$ given by (79).

In Table 6 we make a comparison of the eigenvalues in terms of different heights and widths of the underwater object. Notice that the frequencies remain similar when the height is small and the width is arbitrary. However, as the height $b$ increases the frequencies decrease in a significant way and when the height is tending to 1 , the first eigenvalue is tending to 0 , coinciding with the shallow water regime, see [17]. 
Remark 2. By remark 1, in the case of the rectangle, the eigenvalues equation becomes

$$
-\lambda F^{T} \overline{D E} \mathbf{a}=\pi\left[\left(i-\frac{1}{2}\right) \delta_{i j}\right] \mathbf{a}
$$

with

$$
d_{k r}=\frac{2}{\pi} \int_{-1}^{1} \frac{U_{k-1}\left(x^{\prime}\right) U_{r-1}\left(x^{\prime}\right)}{\sqrt{1-\kappa^{2} x^{\prime 2}}} d x^{\prime} .
$$

We have only computed the eigenvalues for free-end antisymmetric solutions. In a similar fashion one can compute all the other cases, pinned symmetric and antisymmetric and free-end symmetric.

\section{Conclusions}

We have developed a method to compute natural frequencies of a liquid surface bounded by a circular container. The method relies on applying a conformal map into a half-plane, which leads to an integrodifferential equations which can be solved in the natural basis formed by Tchebyshev polynomials. This leads to an eigenvalue problem for matrices that can be approximated by $N \times N$ system whose eigenvalues quickly converge (as $N \rightarrow \infty$ ) to the eigenvalues of the original system. In this way, we computed frequencies for symmetric and antisymmetric eigenfunctions both with pinned and free-end conditions of contact for the free boundary with the container.

A remarkable property of our approach is that all the information for the shape of the container is contained in a matrix $D$ that appears as a factor in our linear systems. This allows automatically to a formulation for containers of any shape that can be conformally mapped into a half-plane. In particular, we use Schwarz-Christoffel map to compute frequencies in a rectangular container with an obstacle in its bottom. Besides illustrating the power of our method, this allowed us to arrive at interesting conclusions on the impact of the obstacle on the frequencies: the width of the obstacle does not play a significant role while the height has a strong impact.

\section{ACKNOWLEDGMENTS}

M.A.Fontelos was partially supported by grant - .

\section{REFERENCES}

[1] T. Alazard, P. Baldi, and D. Han-Kwan. Control of water waves. arXiv preprint arXiv:1501.06366, 2015.

[2] N. Asmar and G. Jones. Applied Complex Analysis with Partial Differential Equations. Prentice Hall, 2002.

[3] T. B. Benjamin and J. C. Scott. Gravity-capillary waves with edge constraints. Journal of Fluid Mechanics, 92(2):241-267, 1979.

[4] J. Bostwick and P. Steen. Coupled oscillations of deformable spherical-cap droplets. part 1. inviscid motions. Journal of Fluid Mechanics, 714:312-335, 2013.

[5] J. Bostwick and P. Steen. Stability of constrained capillary surfaces. Annual Review of Fluid Mechanics, 47, 2015. 
[6] B. Budiansky. Sloshing of liquids in circular canals and spherical tanks. Journal of the Aerospace sciences, 27(3):161-173, 1960.

[7] H.-K. Chang and J.-C. Liou. Long wave reflection from submerged trapezoidal breakwaters. Ocean Engineering, 34(1):185-191, 2007.

[8] W. Craig. An existence theory for water waves and the Boussinesq and KortewegdeVries scaling limits. Communications in Partial Differential Equations, 10(8):7871003, 1985.

[9] M. A. Fontelos, R. Lecaros, J. López-Ríos, and J. H. Ortega. Stationary shapes for 2d water-waves and hydraulic jumps. Journal of Mathematical Physics, 57(8):081520, 2016.

[10] M. A. Fontelos, R. Lecaros, J. López-Ríos, and J. H. Ortega. Bottom detection through surface measurements on water waves. SIAM Journal on Control and Optimization, 55(6):3890-3907, 2017.

[11] E. Godoy, A. Osses, J. H. Ortega, and A. Valencia. Modeling and control of surface gravity waves in a model of a copper converter. Applied Mathematical Modelling, 32(9):1696-1710, 2008.

[12] J. Graham-Eagle. A new method for calculating eigenvalues with applications to gravity-capillary waves with edge constraints. In Mathematical Proceedings of the Cambridge Philosophical Society, volume 94, pages 553-564. Cambridge University Press, 1983.

[13] S. M. Hasheminejad and M. Aghabeigi. Liquid sloshing in half-full horizontal elliptical tanks. Journal of sound and vibration, 324(1-2):332-349, 2009.

[14] H. Hochstadt. Integral equations, volume 91. John Wiley \& Sons, 2011.

[15] H. J. Kim, M. A. Fontelos, and H. J. Hwang. Capillary oscillations at the exit of a nozzle. IMA Journal of Applied Mathematics, 80(4):931-962, 2015.

[16] C. M. Kunkel, R. W. Hallberg, and M. Oppenheimer. Coral reefs reduce tsunami impact in model simulations. Geophysical research letters, 33(23), 2006.

[17] D. Lannes. The water waves problem: mathematical analysis and asymptotics, volume 188. American Mathematical Soc., 2013.

[18] O. S. Madsen and C. C. Mei. The transformation of a solitary wave over an uneven bottom. Journal of Fluid Mechanics, 39(4):781-791, 1969.

[19] J. C. Mason and D. C. Handscomb. Chebyshev polynomials. Chapman and Hall/CRC, 2002 .

[20] V. Nalimov. The Cauchy-Poisson problem. Dynamika Splosh. Sredy, 18:104-210, 1974.

[21] Z. Nehari. Conformal mapping. Courier Corporation, 2012.

[22] H. Nersisyan, D. Dutykh, and E. Zuazua. Generation of $2 \mathrm{~d}$ water waves by moving bottom disturbances. IMA Journal of Applied Mathematics, 80(4):1235-1253, 2014.

[23] J. A. Nicolás. Effects of static contact angles on inviscid gravity-capillary waves. Physics of Fluids, 17(2):022101, 2005.

[24] D. J. Struik. Détermination rigoureuse des ondes irrotationelles périodiques dans un canal à profondeur finie. Mathematische Annalen, 95(1):595-634, 1926.

[25] H. Weinberger. A First Course in Partial Differential Equations: with Complex Variables and Transform Methods. Dover Books on Mathematics. Dover Publications, 2012.

[26] H. Yosihara. Capillary-gravity waves for an incompressible ideal fluid. Kyoto Journal of Mathematics, 23(4):649-694, 1983. 
(M. A. Fontelos) (Corresponding Author) ICMAT-CSIC, C/Nicolás Cabrera, no 13-15 Campus de Cantoblanco, UAM, 28049 Madrid, Spain.

Email address: marco.fontelos@icmat.es

(J. López-Ríos) Escuela de Ciencias Matemáticas y Computacionales, YACHAY TECH, San Miguel de Urcuquí, Hacienda San José S/N, Ecuador

Email address: jlopez@yachaytech.edu.ec 